\title{
Relación entre medidas neuropsicológicas de ejecución y autoinforme de las funciones ejecutivas
}

\section{Relationship between Execution Neuropsychological Measures and Self-report of Executive Functions}

\author{
Kelvin L. Morales-Millán * 1, Yashira Arroyo-Pérez ${ }^{1}$, Maribella González-Viruet ${ }^{1}$, \\ Israel Sánchez-Cardona ${ }^{2}$
}

1 - Universidad Carlos Albizu, San Juan, Puerto Rico.

2 - Kennesaw State University, Georgia, USA.

Introducción

Metodología

Resultados

Discusión

Referencias

Recibido: 15/09/2020 Revisado: 12/10/2020 Aceptado: 15/11/2020

\section{Resumen}

En este estudio se evaluó la relación entre las puntuaciones de funciones ejecutivas (FE) al utilizar medidas de autoinforme versus medidas neuropsicológicas de ejecución. Se examinaron diversos predictores que han sido asociados con las FE para evaluar la convergencia entre las puntuaciones de estas medidas. La muestra consistió en 60 adolescentes varones de entre 14 y 18 años con y sin antecedentes legales. Se utilizaron las puntuaciones del Comprehensive Executive Function Inventory y del DelisKaplan Executive Function System. Los resultados de los análisis de correlación indicaron una relación muy baja y en su mayoría no significativa entre las puntuaciones de ambas medidas. Los resultados del análisis de regresión múltiple indicaron que los predictores de las FE se relacionan de manera disímil con las medidas. No obstante, ambas formas de medir las FE no son excluyentes y pueden ser utilizadas para medir diferentes aspectos de las FE.

Palabras clave: funciones ejecutivas, planificación y organización, flexibilidad cognitiva, control inhibitorio, medidas de autoinforme, medidas neuropsicológicas de ejecución

\begin{abstract}
This study evaluated the relationship between executive function (EF) scores using self-report measures versus performance based neuropsychological measures. Various predictors that have been associated with EFs were examined to assess the convergence of scores for these measures. The sample consisted of 60 male adolescents between 14 and 18 years old with and without legal history. The scores from the Comprehensive Executive Function Inventory and Delis-Kaplan Executive Function System were used. The correlation analysis results indicated a small and mostly non-significant relationship between the scores of both measures. The multiple regression analysis results indicated that the predictors of EF are dissimilarly related to the measures. However, both ways of measuring EF are not exclusive, and it can be useful to use them to measure different EF aspects.
\end{abstract}

Keywords: executive functions, planning and organization, cognitive flexibility, inhibitory control, self-report measures, neuropsychological measures 


\section{Introducción}

Tradicionalmente la evaluación de las funciones ejecutivas (FE) se realiza a través de pruebas neuropsicológicas. Sin embargo, actualmente se han desarrollado instrumentos de autoinforme que exploran el funcionamiento ejecutivo conductual de las personas en la vida cotidiana. Ante las diferentes medidas de evaluación de las FE, se han originado debates sobre cuál de los métodos de evaluación es mejor para la detección de deterioro en el funcionamiento ejecutivo de una persona. Esto representa un problema ya que no existe un consenso acerca de la capacidad de estas medidas para medir con precisión los dominios de las FE. Maldonado-Belmonte (2016) propone que la evaluación de las funciones ejecutivas en la población de niños y adolescentes es compleja por el hecho de que los dominios de las funciones ejecutivas se desarrollan en diferentes momentos $\mathrm{y}$ a distinto ritmo.

Este estudio pretende evaluar si existe alguna relación entre las puntuaciones obtenidas en las pruebas de desempeño o ejecución y el autoinforme que miden las funciones ejecutivas (planificación, flexibilidad cognitiva y organización) de un grupo de jóvenes varones puertorriqueños intervenidos por el Tribunal de Menores de Puerto Rico y un grupo de jóvenes varones puertorriqueños sin antecedentes legales.

\section{Funciones ejecutivas}

A pesar de no haber un consenso en la definición, se ha planteado que el término funciones ejecutivas (FE) hace referencia a un conjunto de habilidades cognitivas superiores y acciones autodirigidas que involucran áreas como la atención selectiva, la inhibición, la flexibilidad cognitiva, la toma de decisiones, la organización y la regula- ción emocional, entre otras (Barkley, 2012).

Para propósitos de este estudio se utilizará el modelo de resolución de problemas desarrollado por Zelazo, Carter, Reznick y Frye (1997). En dicho modelo, se parte de que las FE son un macroconstructo que se extiende a cuatro fases principales de solución de problemas. La primera fase es la presentación del problema, en la que se debe representar el problema de tal manera que sea posible resolverlo. Problemas en esta fase podrían implicar dificultades en habilidades atencionales y en el control inhibitorio. La segunda fase es la planificación, en la que el individuo evalúa su contexto al igual que las opciones que tiene disponibles o que cree lograr para solucionar el problema. La tercera fase es la ejecución e implica tener un plan y lograr traducir el mismo en acción. Por último, se encuentra la fase de evaluación, donde se espera que se evalúe si la solución ejecutada para el problema fue efectiva. Las variables evaluadas en este estudio (planificación, organización, flexibilidad y control inhibitorio) están involucradas en cada una de las cuatro fases para la solución de un problema.

Planificación y organización. La planificación se define como el proceso de identificar posibles opciones, pasos y resultados necesarios para lograr un objetivo específico (Patros, Tarle, Alderson, Lea, \& Arrington, 2019). Por otro lado, el dominio de organización se define como la habilidad de organizar y lograr reconocer la información requerida en un proceso de aprendizaje (Roberts \& Apaza, 2011).

Algunas de las pruebas que se utilizan para medir la planificación y organización son: Tower of London (Shallice, 1982; Krikorian, Bartok, \& Gay, 1994), D-KEFS-prueba de clasificación (Delis, Kaplan, \& Kramer, 2001), Behavioral Rating Inventory of Executive Functioning (BRIEF; Gioia, Isquith, Guy, \& Kenworthy, 2000), Com- 
prehensive Executive Function Inventory (CEFI; Naglieri \& Golstein, 2013), entre otras.

Flexibilidad cognitiva. La flexibilidad cognitiva se define como la habilidad del individuo de cambiar su curso de acción para adaptarse efectivamente a las demandas del ambiente, de una tarea o situación (Rueda, Cómbita, \& Pozuelos, 2016). Algunas pruebas que pueden ser utilizadas para evaluar la flexibilidad cognitiva son: D-KEFS-prueba del trazado (Delis et al., 2001), D-KEFS-prueba de interferencia de color - palabra (Delis et al., 2001), Trail Making Test (Reitan, 1958), Wisconsin Card Sorting Test (Berg, 1948), Test de 5 dígitos (Sedó, 2004), BRIEF (Gioia et al., 2000), CEFI (Naglieri \& Goldstein, 2013), entre otros.

Control inhibitorio. El control inhibitorio es la función que se encarga de suprimir una tendencia automática en una situación dada (Thierry, Glowinski, Goldman-Rakic, \& Christen, 1994). La capacidad de inhibición es necesaria para lograr la negociación de objetivos que compiten entre sí y el retraso de la gratificación inmediata para culminar con los objetivos elegidos a largo plazo (Carlson, Zelazo, \& Faja, 2013). Algunos instrumentos para medir la capacidad de inhibición son: Stroop (Stroop, 1935), Go/No Go (Donders, 1969), Stop signal (Logan \& Cowan, 1984), BRIEF (Gioia et al., 2000), CEFI (Naglieri \& Goldstein, 2013), entre otros.

\section{Problema con la evaluación de las FE}

La falta de unanimidad en la definición de las FE trae consigo problemas para su evaluación. Esto representa una dificultad para comparar las puntuaciones o ejecución del individuo en distintas pruebas, dado que cada una se basa en distintos modelos de las FE. Esta dificultad se complejiza aún más dadas las distintas medidas de evaluación utilizadas para las FE, lo que tiene como consecuencia que los hallazgos de las investigaciones sean inconsistentes entre sí.

En la evaluación neuropsicológica de las funciones ejecutivas existen dos estrategias: a) la aplicación de pruebas de desempeño o ejecución, como son las tareas experimentales creadas para evaluar una función ejecutiva específica en un contexto controlado, y b) las pruebas de autoinforme, que consisten en la perspectiva y observación de las funciones ejecutivas conductuales en las actividades de la vida diaria.

Las medidas de ejecución se utilizan para evaluar el funcionamiento cognitivo (Harvey, 2012). Este ha sido el método utilizado típicamente para evaluar las FE (Comesaña, Stelzer, \& Introzzi, 2017; Vélez-Pastrana et al., 2016). Su administración está estandarizada, por lo que los estímulos se presentan de la misma forma y orden a todos los examinados. La ventaja de una prueba estandarizada es que disminuye la posibilidad de errores durante la administración y corrección, facilita la comparación del evaluado con otros grupos y expone a los evaluados a las mismas condiciones y estímulos. La puntuación está compuesta por el tiempo de las respuestas del individuo y cuán correctas o precisas son las mismas. El problema con este tipo de evaluación es que carece de validez ecológica (Bakar, Taner, Soysal, Karakas, \& Turgay, 2011). La validez ecológica en neuropsicología se refiere a la capacidad del instrumento de predecir comportamientos o problemas en el funcionamiento diario a partir del desempeño en la prueba (Spooner \& Pachana, 2006). Esto sugiere que la evaluación con pruebas de desempeño no refleja la capacidad del paciente para hacer uso de sus habilidades para la solución de problemas reales.

Cabe señalar que muchas de las pruebas 
neuropsicológicas que se utilizan actualmente para medir FE presentan niveles moderados de validez ecológica ya que se utilizan para conocer o predecir la funcionalidad del individuo (García-Molina, Tirapu-Ustárroz, \& Roig-Rovira, 2007). Además, este tipo de instrumentos analizan solamente algunos aspectos de la complejidad de las funciones ejecutivas, obviando otros como lo son los procesos metacognitivos en el contexto del diario vivir o cognición social (Suchy, 2015).

Por otro lado, la administración del autoinforme surge por la necesidad de aumentar la validez ecológica y de valorar el funcionamiento ejecutivo del sujeto evaluando su comportamiento en la vida diaria (Pérez-Salas, Ramos, Oliva, \& Ortega, 2016). En ellas se pretende evaluar la percepción del individuo respecto a destrezas del diario vivir relacionadas con las FE, como lo son el manejo de emociones, el poder planificar y organizar las tareas a realizar durante el día, entre otras. Este tipo de medida permite predecir algún deterioro en las actividades cotidianas, así como el funcionamiento ocupacional (Barkley \& Fischer, 2011). Algunas de sus ventajas son su fácil administración e interpretación y la riqueza de la información recopilada al provenir de primeras manos (Mitchell \& Miller, 2008).

Sin embargo, Paulhus y Vazire (2007) enumeran las siguientes desventajas de los autoinformes: a) la precisión de la información provista se ve afectada por la deseabilidad social y/o respuestas extremas, b) los resultados dependen del conocimiento del individuo y c) la medición puede ser afectada por factores culturales. Hoskin (2012) agregó que dichas medidas tienen una gran dependencia de la honestidad del individuo y pueden ser afectadas por la falta de destrezas de introspección, de entendimiento de la prueba y por el uso de diferentes interpretaciones de las puntuaciones de la escala de valoración. Además, en personas con deterioro cognitivo los autoinfor- mes tienden a perder su utilidad.

Se han realizado varias investigaciones en las cuales se evalúa a las FE en distintos contextos utilizando una o ambas medidas y se ha encontrado que las puntuaciones son inconsistentes entre sí. Por ejemplo, en un estudio realizado por Mitchell y Miller (2008) se buscó evaluar la relación entre las medidas de las funciones ejecutivas y las habilidades funcionales tanto observadas como autoinformadas. Para evaluar las FE, los autores administraron el Delis-Kaplan Executive Function System (D-KEFS), especificamente las pruebas: Trail Making Test 4, Verbal Fluency Test y el Design Fluency Test. En los resultados de dicho estudio se observó que las pruebas mencionadas son más sensibles para detectar deterioro en las funciones ejecutivas que las pruebas de autoinforme de las habilidades de funcionamiento. Esto coincide con lo que han mencionado varios autores sobre la inconsistencia de los resultados para establecer relación entre las medidas de autoinforme y las neuropsicológicas para la evaluación de las FE (Loewenstein et al., 2001; Zanetti, Frisoni, Rozzini, Bianchetti, \& Trabucchi, 1998).

Toplak, West y Stanovich (2013) concluyeron en su estudio que ambas medidas miden distintos aspectos de las FE. Explican que son diferentes en sus procesos de administración, las exigencias al evaluado y las puntuaciones por dominios. Las pruebas de ejecución implican un nivel de estructura más alto de parte del examinador a diferencia de las pruebas de autoinforme, que no requieren de dicho factor. De igual manera, los autores encontraron que existe una asociación de baja a moderada entre los dominios de ambas medidas. Por último, mencionan que, desde una perspectiva teórica de la ciencia cognitiva, ambos constructos evalúan distintos niveles de las FE: las medidas de ejecución miden la eficiencia del procesamiento, mientras que las de autoinforme miden la búsqueda de objetivos personales. 
Estas discrepancias en los artículos mencionados pueden deberse a diferentes factores. Uno de ellos puede ser la diferencia entre las teorías en que se basa cada prueba, es decir, el modelo teórico. Otro, es el hecho de que las pruebas de autoinforme pueden verse influenciadas por distintos factores (como la deseabilidad social, factores culturales, conocimiento del evaluado, entre otros). Todo esto hace relucir la necesidad de seguir evaluando, por un lado, si existe alguna relación entre ambas medidas, y por otro, la capacidad de evaluación de cada una con respecto a las funciones ejecutivas.

Varios autores recomiendan la integración de las pruebas de ejecución o desempeño con autoinformes del funcionamiento ejecutivo para obtener una imagen más completa de las habilidades ejecutivas del individuo (Nęcka, Lech, Sobczyk, \& Śmieja, 2012; Nordvall, Jonsson, \& Stigsdotter-Neely, 2017). Además, la integración de las medidas permite evaluar la asociación entre las dos metodologías de evaluación (Nęcka et al., 2012; Nordvall et al., 2017). Una mejor comprensión de la relación entre ambos instrumentos puede favorecer el desarrollo de procedimientos más efectivos para la evaluación de las funciones ejecutivas. Sin embargo, hasta el momento de revisión solo se encontró un estudio que mide la relación entre las pruebas de ejecución y las medidas autoinforme en adolescentes con problemas legales (Nordvall et al., 2017). No obstante, en Puerto Rico no existen investigaciones de este tipo en jóvenes transgresores. En los estudios antes mencionados los autores se centran en la comparación de las puntuaciones de cada prueba sin tomar en consideración otros de sus factores. Uno de estos factores se relaciona con evaluar si estas discrepancias existen en la relación de cada prueba con los predictores de un buen funcionamiento ejecutivo, tomando en cuenta que ambas medidas son construidas y miden aspectos distintos den- tro de las FE. Es por esto que en este estudio se pretende examinar utilizando ambas medidas $\mathrm{y}$ tomando en cuenta los predictores sociodemográficos de las FE. Se considera que, si las puntuaciones de las FE medidas utilizando autoinformes y pruebas de ejecución se correlacionan entre sí, esto significaría una concordancia sobre el constructo que miden.

Funcionamiento ejecutivo y conductas transgresoras/antisociales

La adolescencia, que comprende el periodo entre la infancia y la edad adulta, está asociada a cambios biológicos, psicológicos, cognitivos y sociales (Blakemore, Burnett, \& Dahl, 2010; Sowell et al., 1999). Durante la adolescencia continúan desarrollándose las regiones de la corteza prefrontal, como la corteza prefrontal dorsomedial y la prefrontal ventral, a causa de la poda sináptica y la mielinización (Nelson, Leibenluft, McClure, \& Pine, 2005). El procesamiento neuronal inmaduro en la corteza frontal y otras regiones corticales y subcorticales conduce a conductas de alto riesgo, la gratificación inmediata y la desregulación emocional (Steinberg, 2010). Mundialmente la transgresión juvenil ha sido un gran desafío, y se ha convertido en un problema mundial de salud pública. La literatura señala que se han intensificado e incrementado las conductas consideradas como faltas por parte de los jóvenes en Puerto Rico y a nivel mundial, comúnmente en varones (Dorna-Pesquera \& Caballero-González, 2010).

Las FE son las capacidades necesarias para la autorregulación, incluida la regulación emocional y social. Cada uno de estos dominios, principalmente la habilidad de tomar decisiones tiene un gran impacto en la conducta juvenil. Los déficits en estas capacidades dan lugar a una infle- 
xibilidad cognitiva que puede resultar en comportamientos inapropiados, incapacidad para planificar y resolver problemas, incapacidad de ver el punto de vista de otra persona, distracción, agresividad, comportamientos impulsivos y juicio social deficiente. Estas alteraciones de las funciones ejecutivas se asemejan a ciertas características de las conductas antisociales y transgresoras. Esto quiere decir que los procesos de las funciones ejecutivas son importantes en el desarrollo de las conductas antisociales y transgresoras (Fuster, 2000; Mesulam, 2002).

Raine (2002) argumentó que existe una teoría de la disfunción prefrontal basada en el crecimiento tardío de la corteza prefrontal que puede ayudar a explicar la transgresión juvenil. Raine (2002) explica que en la adolescencia surge una interacción entre las demandas sociales y el desarrollo de las funciones ejecutivas. Estas demandas sociales producen una sobrecarga en el desarrollo de la corteza prefrontal que, a su vez, podría ocasionar disfunción prefrontal y una falta de control inhibitorio sobre conductas antisociales y violentas durante la adolescencia (Raine, 2002). Según Raine (2002), habrá mayor incidencia de transgresión juvenil en ambientes psicosociales menos estructurados y estables.

\section{Predictores de funcionamiento ejecutivo}

Existen varios predictores de las FE que se encargan de propiciar que dicho desarrollo se continúe dando de manera esperada para la edad del individuo (Zysset et al., 2018). Se ha encontrado que la exposición a eventos traumáticos familiares está relacionada con bajos niveles de funcionamiento ejecutivo (DePrince, Weinzierl, $\&$ Combs, 2009). Con respecto a los factores sociales, la literatura menciona que la exposición a eventos traumáticos impacta en las FE al afectar estructuras y regiones cerebrales (Teicher \& Samson, 2016). Existen diferentes tipos de eventos traumáticos, tales como el abuso físico o sexual, la violencia doméstica, la violencia en la comunidad, los desastres naturales, la muerte repentina de un familiar, entre otros. Las experiencias traumáticas pueden causar una anormalidad en la organización y función del sistema neural del cerebro, lo cual puede impactar algunas capacidades tales como la memoria, el aprendizaje, el autocontrol, la concentración y las FE (Vasterling \& Brailey, 2005; Op den Kelder, Van den Akker, Geurts, Lindauer, \& Overbeek, 2018).

Por último, Rathinabalan y Naaraayan (2018) coinciden en que el sistema familiar es el centro primario de educación y socialización infantil y juvenil. Por tal razón, las dinámicas intrafamiliares juegan un rol esencial en el desarrollo cognitivo, psicológico, físico y social del adolescente (Lugo-Gil \& Tamis-LeMonda, 2008). Algunos estudios mencionan que las relaciones afectivas seguras, los buenos tratos parentales y la estimulación desempeñan un rol fundamental en la organización, el desarrollo y el funcionamiento cerebral temprano (Schore, 2001).

\section{Objetivos}

El propósito de este estudio es evaluar la relación entre las puntuaciones de funciones ejecutivas al utilizar medidas de autoinforme versus medidas neuropsicológicas de ejecución. De igual modo, se examinan diversos predictores como la violencia comunitaria, cantidad de trauma informado y el apoyo familiar para examinar sus relaciones con las FE y la convergencia entre las puntuaciones de las medidas de ejecución y de autoinforme. 


\section{Hipótesis}

H1: Las puntuaciones de FE evaluadas a través de autoinforme y las obtenidas de medidas neuropsicológicas de ejecución se relacionan significativamente.

H2a: La exposición a la violencia comunitaria se relaciona con las FE medidas a través de pruebas neuropsicológicas de ejecución.

H2b: La exposición a la violencia comunitaria se relaciona con las FE evaluadas a través de medidas de autoinforme.

H3a: La cantidad de trauma se relaciona con las FE medidas a través de pruebas neuropsicológicas de ejecución.

H3b: La cantidad de trauma se relaciona con las FE evaluadas a través de medidas de autoinforme. $\mathrm{H} 4 \mathrm{a}$ : El apoyo familiar se relaciona con las FE medidas a través de pruebas neuropsicológicas de ejecución.

H4b: El apoyo familiar se relaciona con las FE evaluadas a través de medidas de autoinforme.

\section{Método}

Participantes

Este estudio consiste en un análisis de datos secundarios recopilados dentro del proyecto titulado Perfil del funcionamiento ejecutivo y aspectos psicosociales de adolescentes varones intervenidos por el Tribunal de Menores de Puerto Rico (Arroyo-Pérez, 2019). La muestra consistió en 60 adolescentes varones de entre 14 y 18 años, reclutados en dos grupos: (a) un grupo de 30 adolescentes varones intervenidos por el Tribunal de Menores, según lo establecido por la Ley de Menores de Puerto Rico (Ley No 88 de 9 de julio de 1986), que se encontraban en libertad condicional o en un programa de desvío y (b) un grupo de comparación compuesto por 30 menores de dife- rentes comunidades que no poseían antecedentes legales. La investigadora principal del estudio autorizó el uso de los datos. El estudio primario fue aprobado por el Institutional Review Board (IRB) de la Universidad Carlos Albizu en San Juan, Puerto Rico. La Tabla 1 muestra las características de los participantes.

\section{Procedimiento}

La muestra del grupo de adolescentes intervenidos por el Tribunal de Menores fue reclutada en una clínica psicológica para niños y adolescentes en Puerto Rico, donde se ofrecían servicios a adolescentes que se encontraban en un programa de desvío o bajo libertad condicional. Los criterios de inclusión para este grupo fueron: 1) ser varón de entre 14 y 18 años; 2) encontrarse bajo libertad condicional o en algún programa de desvío, y 3) poseer un nivel académico de por lo menos sexto grado. La muestra del grupo de jóvenes sin antecedentes legales se reclutó en comunidades de diferentes zonas a lo largo de Puerto Rico. Los criterios de inclusión fueron: 1) ser varón y tener entre 14 y 18 años; 2) no tener antecedente legal previo, y 3 ) tener un nivel académico de por lo menos sexto grado. Para ambos grupos se consideraron los siguientes criterios de exclusión: 1) presentar una condición mental severa relacionada con esquizofrenia, epilepsia o disfunción intelectual, y 2) haber sufrido de algún trauma en la cabeza que haya terminado en pérdida del conocimiento.

Los jóvenes, que participaron de manera voluntaria, firmaron la hoja de asentimiento, luego de solicitar el consentimiento informado de su encargado o tutor legal. Posteriormente, se procedió a completar la hoja de datos personales y a la administración de la batería de pruebas. Las pruebas fueron administradas en la Clínica de Ni- 
Morales-Millán, et. al., Evaluar, 2021, 21(1), 60-72

Tabla 1

Características sociodemográficas de la muestra.

\begin{tabular}{lccc}
\hline & Tribunal & No Tribunal \\
\hline Participantes $(n)$ & 30 & 30
\end{tabular}

Edad

$\operatorname{Media} \pm \mathrm{DE}$

Servicios de salud mental

Psicológicos

$19(63 \%)$

$12(40 \%)$

Psicológicos después del tribunal o en la actualidad

$30(100 \%)$

$21(70 \%)$

$30(100 \%)$

Diagnóstico salud mental

$17.1 \pm .91$
$17.1 \pm .91$

$11(36 \%)$

$3(10 \%)$

$11(36 \%)$

Diagnóstico salud mental

Relacionados a sustancias

$26(86 \%)$

$17(56 \%)$

$5(45 \%)$

$15(50 \%)$

$9(81 \%)$

Específico del aprendizaje

Destructivos, del control de los impulsos y de la conducta no especificado

$12(40 \%)$

Historial autolesión

$7(23 \%)$

Ansiedad

$7(23 \%)$

$15(50 \%)$

$9(30 \%)$

$17(56 \%)$

$3(10 \%)$

$25(83 \%)$

$10(33 \%)$

$19(63 \%)$

$2(6.7 \%)$

Antecedente legal

Primera intervención por tribunal

$21(70 \%)$

Varias intervenciones por el tribunal

$9(30 \%)$

Tipo de faltas
Uso de sustancias
$27(90 \%)$
Venta de sustancias controladas
$9(30 \%)$
Intimidación y amenaza
$6(20 \%)$
Robo con arma
$5(16 \%)$ 
Tabla 1 (Cont.)

Características sociodemográficas de la muestra.

Tribunal

Tipo de trauma
No Tribunal

$29(96 \%)$

$26(86 \%)$

$23(76 \%) \quad 18(63 \%)$

$22(73 \%)$

$11(36 \%)$

$15(50 \%)$

$11(36 \%)$

Nota. DE: desviación estándar.

ños y Adolescentes especializada en adolescentes con uso de sustancias y problemas legales y en las oficinas privadas de una profesional que trabajaba con adolescentes sin problemas legales. La participación tomó aproximadamente tres horas, con 15 minutos de tiempo libre para una merienda nutritiva. El ambiente de la administración de los instrumentos fue confidencial, con buena iluminación, temperatura adecuada, asientos cómodos, libre de otros estímulos y de distractores (ruidos e interrupciones) que pudieran afectar la participación.

\section{Instrumentos}

\section{Comprehensive Executive Function Inventory} (CEFI, Naglieri \& Goldstein, 2013). El Inventario Comprensivo de Funciones Ejecutivas (CEFI, por sus siglas en inglés) es una prueba para evaluar las FE en individuos desde los 5 hasta los 18 años. El CEFI pretende evaluar el comportamiento del individuo en las últimas cuatro semanas. El mismo contiene formularios para ser contestados por padres, y maestros o el mismo individuo. La prueba está compuesta por 100 ítems en una escala tipo Likert de seis puntos, divididos en 9 escalas: atención, regulación emocional, organización, flexibilidad, planificación, automo- nitoreo, iniciación, memoria de trabajo y control inhibitorio. La administración tiene una duración de aproximadamente 15 minutos. El coeficiente de confiabilidad interna de la prueba fluctuó entre .97 y .99 (Naglieri \& Goldstein, 2013). Por otro lado, la confiabilidad interna entre las nueve escalas fue .77 a .86. Las escalas que se utilizarán en este estudio son las siguientes: control inhibitorio (10 ítems), flexibilidad cognitiva ( 7 ítems), planificación y organización (10 ítems).

\section{Delis-Kaplan Executive Function System (Delis} et al., 2001). El Delis-Kaplan Executive Function System (D-KEFS) es una prueba estandarizada compuesta por nueve subpruebas para evaluar las FE desde los 8 hasta los 89 años de edad. Las subpruebas son: Prueba del Trazado, Prueba de Fluidez Verbal, Prueba de Interferencia de Color-Palabra, Prueba de Clasificación, Prueba de las 20 Preguntas, Prueba del Contexto de la Palabra, Prueba de la Torre, Prueba de fluidez de Diseño y Prueba de Proverbio. Cada subprueba puede ser administrada como única medida de evaluación de manera individual o grupal. En este estudio se utilizaron las siguientes pruebas:

1. DKEFS- Prueba del Trazado: Esta prueba mide las funciones de flexibilidad y control inhibitorio. Se utilizaron las siguientes condiciones: secuencia de números, 
secuencia de letras e intercambio de número y letra. La consistencia interna de la prueba se encuentra entre .69 y .79. (Delis et al., 2001).

2. D-KEFS-Prueba de Interferencia de Color-Palabra: Consiste en evaluar la flexibilidad cognitiva y el control inhibitorio. Se utilizó la subprueba de alternar entre mencionar el color de la tinta y leer la palabra incongruente. La confiabilidad interna entre las subtareas fue de entre .69 y .75 .

3. D-KEFS-Prueba de Clasificación: evalúa la capacidad de planificación y organización. Dicha prueba consiste en dos tareas: a) clasificación libre (clasificar las cartas en dos grupos de forma espontánea en estímulos verbales y no verbales); y b) reconocimiento de categorías (organizar las cartas en dos grupos a partir de ocho posibles criterios para que luego el evaluado describa e identifique los criterios utilizados en la organización). La tarea de clasificación libre obtuvo una consistencia interna de entre .55 y .82 ; mientras que para la tarea de reconocimiento de categorías se encuentra entre .72 a .74.

Violencia comunitaria. La violencia comunitaria se examinó a través de la siguiente pregunta: ¿Has experimentado violencia comunitaria en algún momento de tu vida (por ejemplo, tiroteo, asalto, venta de drogas)? Los participantes contestaron utilizando dos alternativas de respuesta $(0=N o ; 1=S i)$.

Cantidad de Trauma. Para examinar cuán expuestos habían estado los menores a experiencias extremadamente estresantes, se utilizó una lista de 20 eventos adversos. Algunos de los eventos adversos que se preguntaron fueron los siguientes: maltrato físico, negligencia, maltrato emocional, abandono, acoso escolar, abuso sexual, remoción del hogar, entre otras experiencias traumáticas. Los participantes utilizaron una escala dicotómica $(0=N o ; 1=S i)$ para informar si habían experimentado alguna de estas situaciones a lo largo de su vida. Para calcular la cantidad de trauma a la que habían sido expuestos se sumaron todas las respuestas otorgadas a los 20 eventos adversos.

Apoyo familiar. Para examinar la percepción de apoyo familiar se utilizó un cuestionario de 13 ítems desarrollado para la investigación primaria (Arroyo-Pérez, 2019). Algunos de los ítems incluidos en el cuestionario son: Mi familia es unida, Mi familia me escucha y Me siento amado por mi familia. Todos los ítems se responden por medio de una escala Likert de 7 puntos (de $0=N u n$ ca a 6 -Siempre). A mayor puntuación, mayor percepción de apoyo familiar. Este instrumento mostró buena consistencia interna $(\alpha=.87)$.

\section{Análisis de datos}

Para evaluar si existe relación entre las puntuaciones de las medidas de ejecución y de autoinforme de FE se utilizó un análisis de correlación producto-momento de Pearson. Además, para examinar la relación de algunos de los predictores de las FE definidos por la literatura (p. ej., violencia comunitaria, cantidad de trauma, apoyo familiar, etc.) se realizó un análisis de regresión lineal múltiple. Se utilizó el coeficiente estandarizado $(\mathbb{R})$ para identificar la relación de cada predictor con las puntuaciones de las medidas de ejecución y de autoinforme de FE. Además, se calculó el coeficiente de determinación $\left(R^{2}\right)$ de cada modelo como medida del tamaño del efecto. Todos los análisis se realizaron con el programa SPSS v.26 (IBM Corp., 2019). 


\section{Resultados}

La Tabla 2 muestra los resultados de las correlaciones entre las puntuaciones de las subpruebas que miden las FE de planificación y organización, flexibilidad cognitiva y control inhibitorio, evaluadas a través de pruebas neuropsicológicas de ejecución y de autoinforme. Se encontraron relaciones fuertes (coeficiente $r$ de .49 a .65) entre las puntuaciones de las distintas áreas de la prueba de Delis-Kaplan (prueba neuropsico- lógica de ejecución). De igual modo, las distintas subpruebas del CEFI (medida de autoinforme) se relacionaron significativamente (coeficiente $r$ de .59 a .63). Al examinar la relación entre las puntuaciones de las medidas de neuropsicológicas de ejecución y las de autoinforme, se encontraron algunas relaciones significativas entre medidas de distintas dimensiones de las funciones ejecutivas. Por ejemplo, se encontró que las puntuaciones de las subescalas del CEFI para planificación y organización se relacionaban significativamente y de

Tabla 2

Correlaciones entre las puntuaciones de las funciones ejecutivas medidas a través de pruebas neuropsicológicas de ejecución y de autoinforme.

\begin{tabular}{|c|c|c|c|c|c|c|c|c|c|c|c|c|c|c|c|}
\hline & $\mathbf{M}$ & DE & 1 & 2 & 3 & 4 & 5 & 6 & 7 & 8 & 9 & 10 & 11 & 12 & 13 \\
\hline $\begin{array}{l}\text { 1. D-KEFS Sorting Test: Condi- } \\
\text { tion 1: Confirmed Correct Sort }\end{array}$ & 6.42 & 2.75 & & & & & & & & & & & & & \\
\hline $\begin{array}{l}\text { 2. D-KEFS Sorting Test: Condi- } \\
\text { tion 1: Free Sorting Description } \\
\text { Score }\end{array}$ & 6.63 & 3.09 & $.96^{* *}$ & & & & & & & & & & & & \\
\hline $\begin{array}{l}\text { 3. D-KEFS Sorting Test: Condi- } \\
\text { tion 2: Sort Recognition }\end{array}$ & 7.38 & 3.32 & $.89 * *$ & $.90 * *$ & & & & & & & & & & & \\
\hline $\begin{array}{l}\text { 4. D-KEFS -Trail Making Test: } \\
\text { Number Sequencing }\end{array}$ & 9.53 & 2.69 & $.48 * *$ & $.51 * *$ & $.51^{* *}$ & & & & & & & & & & \\
\hline $\begin{array}{l}\text { 5. D-KEFS -Trail Making Test: } \\
\text { Letter Sequencing }\end{array}$ & 6.75 & 4.13 & $.68^{* *}$ & $.68^{* *}$ & $.69^{* *}$ & $.72 * *$ & & & & & & & & & \\
\hline $\begin{array}{l}\text { 6. D-KEFS -Trail Making Test: } \\
\text { Number-Letter Switching }\end{array}$ & 7.35 & 3.17 & $.60 * *$ & $.58 * *$ & $.63^{* *}$ & $.67 * *$ & $.75 * *$ & & & & & & & & \\
\hline $\begin{array}{l}\text { 7. D-KEFS-Color Test: Inhibi- } \\
\text { tion/Switching }\end{array}$ & 8.13 & 3.79 & $.54 * *$ & $.53 * *$ & $.51^{* *}$ & $.49 * *$ & $.57 * *$ & $.65^{* *}$ & & & & & & & \\
\hline 8. CEFI_Organización & 95 & 13.7 & .21 & .17 & .15 & $.29 *$ & $.33 * *$ & $.33 * *$ & .19 & & & & & & \\
\hline 9. CEFI_Planificación & 90.2 & 15.4 & .20 & .19 & .23 & $.37 * *$ & $.40 * *$ & $.42 * *$ & .21 & $.77 * *$ & & & & & \\
\hline 10. CEFI_Flexibilidad Cognitiva & 100 & 14.5 & .11 & .09 & .07 & .24 & .19 & .18 & .09 & $.57 * *$ & $.60 * *$ & & & & \\
\hline 11. CEFI_Control Ihibitorio & 90.4 & 19.9 & $.29 *$ & $.27 *$ & $.27 *$ & $.44 * *$ & $.33 * *$ & $.31^{*}$ & .21 & $.59 * *$ & $.61 * *$ & $.63 * *$ & & & \\
\hline 12. Violencia Comunitaria & - & - & -.21 & -.25 & $-.28^{*}$ & -.10 & $-.35 * *$ & $-.27 *$ & -.11 & -.17 & -.14 & -.1 & -.04 & & \\
\hline 13. Cantidad de Trauma & 6.82 & 3.78 & $-.34 * *$ & $-.34 * *$ & $-.38 * *$ & $-.36^{* *}$ & $-.34 * *$ & $-.35 * *$ & -.22 & -.08 & -.17 & -.16 & -.15 & $.63^{* *}$ & \\
\hline 14. Apoyo Familiar & 53.3 & 15 & .10 & .05 & .01 & .12 & 0 & .01 & 0 & .15 & .24 & $.36^{* *}$ & .22 & -.03 & $-.50 * *$ \\
\hline
\end{tabular}

Nota. ${ }^{* *} p<.01$ ( 2 colas $) ;{ }^{*} p<.05$ ( 2 colas $)$. 
Tabla 3

Resultados de regresión para los indicadores de Planificación y Organización con medidas de neuropsicológicas de ejecución y de autoinforme.

\begin{tabular}{|c|c|c|c|c|c|c|c|c|c|c|c|c|c|}
\hline \multicolumn{14}{|c|}{ Planificación y Organización } \\
\hline \multicolumn{14}{|c|}{ Medidas neuropsicológicas de ejecución } \\
\hline \multirow{3}{*}{$\begin{array}{l}\text { Violencia comu- } \\
\text { nitaria }\end{array}$} & \multicolumn{4}{|c|}{$\begin{array}{c}\text { D-KEFS Sorting Test: } \\
\text { Condition 1: Confirmed Correct Sort }\end{array}$} & \multicolumn{5}{|c|}{$\begin{array}{l}\text { D-KEFS Sorting Test: } \\
\text { Condition 1: Free Sorting Description }\end{array}$} & \multicolumn{4}{|c|}{$\begin{array}{c}\text { D-KEFS Sorting Test: } \\
\text { Condition 2: Sort Recognition }\end{array}$} \\
\hline & $\beta$ & SE & $p$ & {$[95 \% \mathrm{IC}]$} & $\beta$ & $\mathrm{SE}$ & $\stackrel{p}{p}$ & {$[95 \%$ IC } & & $\beta$ & SE & $p$ & {$[95 \% \mathrm{IC}]$} \\
\hline & .05 & .98 & .76 & {$[-1.68,2.28]$} & .03 & 1.09 & .85 & {$[-2.11,2.4$} & & .06 & 1.14 & .73 & {$[-1.86,2.65]$} \\
\hline $\begin{array}{l}\text { Cantidad de Trau- } \\
\text { ma }\end{array}$ & -.43 & .15 & .04 & {$[-.59,-.02]$} & -.45 & .16 & .03 & {$[-.66,-.06$} & & -.55 & .17 & .01 & {$[-.81,-.134]$} \\
\hline \multirow{2}{*}{$\begin{array}{l}\text { Apoyo Familiar } \\
\text { Adjusted } \mathrm{R}^{2}\end{array}$} & -.11 & .02 & .47 & {$[-.08, .05]$} & -.17 & .03 & .28 & {$[-.10, .03$} & & -.25 & .03 & .10 & {$[-.12, .018]$} \\
\hline & .08 & & & & .09 & & & & & .14 & & & \\
\hline \multirow[t]{2}{*}{$\mathrm{R}^{2}$} & .13 & & & & .14 & & & & & .18 & & & \\
\hline & \multicolumn{4}{|c|}{$\mathrm{F}(3,56)=2.67, p=.05$} & & $3,56)=$ & $=3.038,1$ & $p=.036$ & & \multicolumn{4}{|c|}{$\mathrm{F}(3,56)=4.34, p=.008$} \\
\hline \multicolumn{14}{|c|}{ Planificación y Organización } \\
\hline \multicolumn{14}{|c|}{ Medidas de autoinforme } \\
\hline & \multicolumn{6}{|c|}{ CEFI_Organización } & \multicolumn{7}{|c|}{ CEFI_Planificación } \\
\hline \multirow{2}{*}{$\begin{array}{l}\text { Violencia comu- } \\
\text { nitaria }\end{array}$} & $\beta$ & $\mathrm{SE}$ & $p$ & \multicolumn{3}{|c|}{$[95 \% \mathrm{IC}]$} & $\beta$ & $\mathrm{SE}$ & $p$ & & \multicolumn{3}{|c|}{$[95 \% \mathrm{IC}]$} \\
\hline & -.34 & 5.00 & .06 & \multicolumn{3}{|c|}{$[-18.63,1.30]$} & -.18 & 5.63 & .31 & & \multicolumn{3}{|c|}{$[-16.88,6.69]$} \\
\hline $\begin{array}{l}\text { Cantidad de Trau- } \\
\text { ma }\end{array}$ & .27 & .76 & .20 & \multicolumn{3}{|c|}{$[-.78,2.85]$} & .08 & .86 & .68 & & \multicolumn{3}{|c|}{$[-1.19,1.94]$} \\
\hline \multirow{2}{*}{$\begin{array}{l}\text { Apoyo Familiar } \\
\text { Adjusted } \mathrm{R}^{2}\end{array}$} & .27 & .15 & .09 & \multicolumn{3}{|c|}{$[-.01, .567]$} & .28 & .16 & .08 & & \multicolumn{3}{|c|}{$[-.011, .603]$} \\
\hline & .03 & & & & & & .03 & & & & & & \\
\hline \multirow[t]{2}{*}{$\mathrm{R}^{2}$} & .08 & & & & & & .08 & & & & & & \\
\hline & \multicolumn{6}{|c|}{$\mathrm{F}(3,56)=1.63, p=.193$} & \multicolumn{7}{|c|}{$\mathrm{F}(3,56)=1.68, p=.182$} \\
\hline
\end{tabular}

forma moderada (coeficiente $r$ de .29 a .42) con las puntuaciones del D-KEFS-Trail Making Test: Number Sequencing (flexibilidad cognitiva), D-KEFS-Trail Making Test: Letter Sequencing (flexibilidad cognitiva), D-KEFS-Trail Making Test: Number-Letter Switching y D-KEFS-Color Test: Inhibition/Switching (control inhibitorio). De igual forma, la subprueba que examina el control inhibitorio en el CEFI tuvo una relación significativa con las puntuaciones de las subpruebas del Delis Kaplan que examinan planificación y organización (D-KEFS Sorting Test: Condition 1: Confirmed Correct Sort; D-KEFS Sorting Test: Condition 1: Free Sorting Description Score;
D-KEFS Sorting Test: Condition 2: Sort Recognition), flexibilidad cognitiva (D-KEFS -Trail Making Test: Number Sequencing; D-KEFS -Trail Making Test: Letter Sequencing) y control inhibitorio (D-KEFS -Trail Making Test: Number-Letter Switching). Estas correlaciones van de .27 a .44 .

Se realizó un modelo de regresión múltiple para examinar si existe relación entre la exposición a la violencia comunitaria, la cantidad de eventos traumáticos informados y el apoyo familiar, y las puntuaciones de las subpruebas que miden las funciones ejecutivas de planificación y organización, flexibilidad cognitiva y control in- 
hibitorio. En la Tabla 3 se presentan los resultados del modelo de regresión múltiple para las medidas de planificación y organización en pruebas neuropsicológicas de ejecución y de autoinforme. Los resultados indican que solo la cantidad de trauma tuvo un efecto negativo y significativo con respecto a las puntuaciones de las subpruebas de la prueba Delis-Kaplan que examinan planificación y organización: D-KEFS Sorting Test: Condition 1: Confirmed Correct Sort $(\beta=-.43$, $p<.05)$, Sorting Test: Condition 1: Free Sorting
Description Score $(\beta=-.45, p<.05)$, D-KEFS Sorting Test: Condition 2: Sort Recognition ( $\beta=$ $-.55, p<.05)$. No se encontró ninguna relación significativa entre la exposición a la violencia, la cantidad de eventos traumáticos reportados y el apoyo familiar por una parte y las puntuaciones de las FE de planificación y organización medidas con pruebas de autoinforme por otra parte.

En la Tabla 4 se presentan los resultados relacionados con las medidas de flexibilidad cognitiva. Los resultados indican que la cantidad de

Tabla 4

Resultados de regresión para los indicadores de Flexibilidad Cognitiva y Control Inhibitorio con medidas neuropisológicas de ejecución y de autoinforme.

\begin{tabular}{|c|c|c|c|c|c|c|c|c|c|c|c|c|}
\hline \multicolumn{13}{|c|}{ Flexibilidad cognitiva } \\
\hline & \multicolumn{4}{|c|}{$\begin{array}{l}\text { D-KEFS -Trail Making Test: } \\
\text { Number Sequencing }\end{array}$} & \multicolumn{4}{|c|}{$\begin{array}{l}\text { D-KEFS -Trail Making Test: } \\
\text { Letter Sequencing }\end{array}$} & \multicolumn{4}{|c|}{ CEFI_Flexibilidad Cognitiva } \\
\hline & $\beta$ & SE & $p$ & {$[95 \% \mathrm{IC}]$} & $\beta$ & SE & $p$ & {$[95 \% \mathrm{IC}]$} & $\beta$ & SE & $p$ & {$[95 \% \mathrm{IC}]$} \\
\hline $\begin{array}{l}\text { Violencia } \\
\text { comunitaria }\end{array}$ & .30 & .92 & .08 & {$[.13,3.07]$} & -.13 & 1.44 & .44 & {$[-.50,1.58]$} & -.33 & 5.00 & .06 & {$[-19.17,-.70]$} \\
\hline $\begin{array}{l}\text { Cantidad de } \\
\text { Trauma }\end{array}$ & -.65 & .14 & .00 & {$[-.75,-.15]$} & -.34 & .22 & .09 & {$[-.79, .01]$} & .29 & .76 & .14 & {$[-.39,2.51]$} \\
\hline $\begin{array}{l}\text { Apoyo Fami- } \\
\text { liar }\end{array}$ & -.19 & .02 & .21 & {$[-.08, .02]$} & -.17 & .04 & .27 & {$[-.14, .06]$} & .50 & .15 & .00 & {$[.18, .78]$} \\
\hline Adjusted $\mathrm{R}^{2}$ & .13 & & & & .12 & & & & .14 & & & \\
\hline \multirow[t]{2}{*}{$\mathrm{R}^{2}$} & .18 & & & & .16 & & & & .185 & & & \\
\hline & \multicolumn{4}{|c|}{$\mathrm{F}(3,56)=4.183, p=.010$} & \multicolumn{4}{|c|}{$\mathrm{F}(3,56)=3.72, p=.016$} & \multicolumn{4}{|c|}{$\mathrm{F}(3,56)=4.247, p=.009$} \\
\hline
\end{tabular}

Tabla 5

Resultados de regresión para los indicadores de Control Inhibitorio con medidas neuropsicológica de ejecución y de autoinforme.

\begin{tabular}{|c|c|c|c|c|c|c|c|c|c|c|c|c|}
\hline \multicolumn{13}{|c|}{ Control Inhibitorio } \\
\hline & \multicolumn{4}{|c|}{$\begin{array}{l}\text { D-KEFS -Trail Making Test: Num- } \\
\text { ber-Letter Switching }\end{array}$} & \multicolumn{4}{|c|}{$\begin{array}{l}\text { D-KEFS-Color Test: Inhibition/ } \\
\text { Switching }\end{array}$} & \multicolumn{4}{|c|}{ CEFI_Control Inhibitorio } \\
\hline & $\beta$ & SE & $p$ & {$[95 \% \mathrm{IC}]$} & $\beta$ & SE & $p$ & {$[95 \% \mathrm{IC}]$} & $\beta$ & SE & $p$ & {$[95 \% \mathrm{IC}]$} \\
\hline $\begin{array}{l}\text { Violencia } \\
\text { comunitaria }\end{array}$ & .03 & 1.12 & .86 & {$[-2.03,2.58]$} & .13 & 1.32 & .45 & {$[-1.68,4.06]$} & -.01 & 7.38 & .97 & {$[-14.38,15.30]$} \\
\hline $\begin{array}{l}\text { Cantidad de } \\
\text { Trauma }\end{array}$ & -.48 & .17 & .02 & {$[-.72,-.06]$} & -.41 & .213 & .05 & {$[-.83, .01]$} & -.04 & 1.13 & .82 & {$[-.25,2.09]$} \\
\hline $\begin{array}{l}\text { Apoyo Fami- } \\
\text { liar }\end{array}$ & -.23 & .03 & .60 & {$[-.11, .04]$} & -.20 & .04 & .21 & {$[-.12, .04]$} & .19 & .22 & .60 & {$[-.17, .69]$} \\
\hline Adjusted $\mathrm{R}^{2}$ & .11 & & & & .028 & & & & .000 & & & \\
\hline \multirow[t]{2}{*}{$\mathrm{R}^{2}$} & .16 & & & & .077 & & & & .051 & & & \\
\hline & \multicolumn{4}{|c|}{$\mathrm{F}(3,56)=3.56, p=.020$} & \multicolumn{4}{|c|}{$\mathrm{F}(3,56)=1.557, p=.210$} & \multicolumn{4}{|c|}{$\mathrm{F}(3,56)=1.00, p=.400$} \\
\hline
\end{tabular}


eventos traumáticos se relaciona con las puntuaciones de la subprueba de D-KEFS -Trail Making Test: Number Sequencing para flexibilidad cognitiva $(\beta=-.65, p<.05)$. En el caso de la subprueba D-KEFS -Trail Making Test: Letter Sequencing, aunque el resultado no fue significativo, se encontró una relación moderada $(\beta=-.34, p=.09)$. Un resultado similar se observa al examinar el efecto de la violencia comunitaria en la flexibilidad cognitiva evaluada a través del D-KEFS-Trail Making Test: Number Sequencing. Se encontró un efecto moderado, aunque no estadísticamente significativo $(\beta=.30, p=.08)$. Al considerar las relaciones con las puntuaciones de las pruebas de autoinforme encontramos que la violencia comunitaria tiene un efecto moderado, aunque no estadísticamente significativo con la flexibilidad cognitiva $(\beta=-.33, p=.06)$. Además, se encontró que el apoyo familiar se relaciona significativamente con la flexibilidad cognitiva evaluada a través de autoinforme $(\beta=.50, p<.05)$.

Por último, la Tabla 5 presenta los resultados relacionados con las medidas de control inhibitorio. Al examinar las relaciones con las puntuaciones de las pruebas neuropsicológicas de ejecución encontramos que solo la cantidad de trauma mostró una relación significativa al evaluarse con la subprueba D-KEFS -Trail Making Test: Number-Letter Switching $(\beta=-.48, p<.05)$. El resultado con las puntuaciones de la subprueba D-KEFS-Color Test: Inhibition/Switching, aunque no fue significativo, refleja un efecto moderado $(\beta=-.41, p=.05)$. No se encontró ninguna relación significativa entre la exposición a la violencia, la cantidad de eventos traumáticos informada y el apoyo familiar por una parte y las puntuaciones de control inhibitorio evaluado a través de pruebas de autoinforme por la otra.

La prueba Shapiro-Wilk para el análisis de normalidad indica que las puntuaciones de algunas variables no se distribuyen normalmente (p. ej., violencia comunitaria, cantidad de trauma, violencia comunitaria, D-KEFS Sorting Test: Condition 1: Confirmed Correct Sort, CEFI_Organización, D-KEFS -Trail Making Test: Number Sequencing, D-KEFS -Trail Making Test: Letter Sequencing, D-KEFS -Trail Making Test: Number-Letter Switching, D-KEFS-Color Test: Inhibition/Switching). Por otro lado, los indicadores de tolerancia (violencia comunitaria $=.48$; cantidad de trauma $=.36$; apoyo familiar $=.60$ ) son mayores a .20, mientras que los indicadores de factor de inflación de varianza (Variance Inflation factor [VIF], en inglés) fueron todos menores de 10 (violencia comunitaria $=2.05$; cantidad de trauma $=2.75$; apoyo familiar $=1.65$ ) lo que indica que no existen problemas de multicolinealidad en los análisis de regresión. Con el objetivo de ofrecer una mayor precisión en los estimados debido a la limitación de la muestra y la no normalidad de algunas variables, se calcularon intervalos de confianza al 95\% con 1000 muestras bootstrapped en todos los análisis (Field, 2018). Estos intervalos permiten una comprensión más clara y comparable de la proporción de la varianza explicada (Bologna, 2014). Todos los análisis se realizaron con el programa SPSS v.26 (IBM Corp., 2019).

\section{Discusión}

Este estudio tenía como propósito explorar si existe una relación entre las puntuaciones de pruebas de autoinforme y de ejecución para la evaluación de las FE, así como conocer las relaciones con diversos predictores del funcionamiento ejecutivo (p. ej., la cantidad de eventos traumáticos, el apoyo familiar y la violencia comunitaria). De la literatura revisada se desprende que existen ciertas dificultades u obstáculos al evaluar las FE utilizando estas dos medidas, es- 
pecíficamente al comparar las puntuaciones. Algunos autores han encontrado discrepancias entre estas puntuaciones (Nordvall et al., 2017; Toplak et al., 2013). Para verificar estos hallazgos se evaluó la relación entre ellas.

Las investigaciones señalan que la ausencia de correlación entre las medidas de autoinformes y pruebas neuropsicológicas se debe a que evalúan aspectos diferentes de las funciones ejecutivas mencionadas (Toplak et al., 2013; Suchy, 2015). Por lo tanto, los resultados de ambas medidas no deben ser interpretados como equivalentes o intercambiables. Esto puede implicar que ambas medidas son esenciales para realizar una evaluación completa, ya que permiten al evaluador desarrollar recomendaciones prescriptivas, específicas y holísticas (emocionales, conductuales y cognitivas) en relación con el funcionamiento ejecutivo.

Las únicas relaciones significativas encontradas fueron aquellas que involucran a las variables del mismo constructo. Se encontró relación entre las funciones ejecutivas planificación y organización y flexibilidad cognitiva tanto en las medidas de autoinforme como en las neuropsicológicas. Esto puede deberse a que la habilidad de planificación se compone de otros procesos como la memoria y la flexibilidad cognitiva. El control inhibitorio y la flexibilidad cognitiva son componentes importantes en las tareas relacionadas a la planificación, por ejemplo, para determinar si los participantes toman tiempo para pensar en lugar de actuar impulsivamente (Asato, Sweeney, \& Luna, 2006). Cualquier tarea que requiera manejo del tiempo, memoria y flexibilidad en el pensamiento será un desafío para las personas con limitaciones en la capacidad de planificación.

La literatura menciona que la exposición a eventos traumáticos impacta en las FE al afectar estructuras y regiones cerebrales (Teicher \& Samson, 2016). Por ejemplo, las regiones cere- brales más afectadas en los casos de jóvenes que sufren maltrato son la corteza prefrontal y la corteza orbitofrontal, que están relacionadas con las FE (Teicher \& Samson, 2016). Se encontró una relación estadísticamente significativa y negativa entre cantidad de eventos traumáticos y algunas funciones ejecutivas medidas por las pruebas neuropsicológicas. Estos resultados indican que, a mayor cantidad de eventos traumáticos, menor es el desempeño en las medidas neuropsicológicas que evalúan las funciones ejecutivas control inhibitorio, planificación y organización y flexibilidad cognitiva. Sin embargo, es importante tomar en cuenta que no podemos obtener conclusiones sólidas por la limitación de la muestra.

\section{Conclusión}

La utilidad de una de estas medidas sobre otra es un aspecto que se debe seguir investigando. Los resultados de esta investigación indican que el uso de un tipo de prueba no excluye el de la otra, por lo que parecería ser de mayor utilidad usar ambas medidas para medir las FE. Esto puede estar sugiriendo que ambas medidas examinan aspectos diferentes de las FE. El autoinforme mide el área conductual y emocional de la vida del sujeto y las pruebas neuropsicológicas evalúan los factores metacognitivos de las funciones en un ambiente con condiciones estructuradas. Estos resultados nos señalan que las FE son complejas y se componen de diferentes niveles de procesos metacognitivos, reguladores emocionales y reguladores conductuales.

No obstante, para futuras investigaciones se recomienda utilizar otro modelo de las funciones ejecutivas en donde se identifiquen los diferentes procesos que interactúan en las mismas, tanto las funciones de orden superior como los componentes emocionales y conductual. Por otro 
lado, se sugiere que se identifiquen más variables predictoras para ser utilizadas en la evaluación de estas dos medidas. Aunque los resultados de este estudio comparten algunas semejanzas con lo hallado en la literatura, sirven para propiciar otros estudios con una muestra mayor, al igual que una mayor selección de pruebas de ambas medidas. Una vez incorporadas otras pruebas y variables se podrá tener una visión más amplia y clara sobre la utilidad de ambas medidas.

Durante el progreso de este estudio se pudieron identificar varias limitaciones. Al ser un análisis de datos secundarios, se analizaron los datos recopilados en la investigación primaria. Esto presenta varias limitaciones en cuanto a las medidas de ejecución y autoinforme de las FE disponibles para examinar. De igual modo, al considerar los predictores, solo se pudieron utilizar en los análisis algunos de los mencionados por la literatura. Cabe señalar que en el estudio principal se demostró que existen diferencias en las puntuaciones entre el grupo de jóvenes transgresores y el grupo de jóvenes de la comunidad sin problemas legales. Durante la revisión de literatura se encontraron otras variables que podrían ser consideradas, las cuales no se pudieron explorar en la presente investigación. Algunas de estas variables son el estatus socioeconómico y el tipo de crianza.

Es importante mencionar que, al ser un análisis de datos secundarios, la muestra de adolescentes varones de entre 14 y 18 años disponible fue pequeña, lo que limita la posibilidad de generalización de los resultados. Estudios posteriores deben examinar diferencias o convergencias en la medición de las funciones ejecutivas utilizando muestras más heterogéneas en género y edad. De igual modo, la no significancia de algunos predictores con relación a las medidas de funciones ejecutivas (de desempeño/ejecución y autoinforme) puede deberse a la muestra limitada con la que contó el estudio. Futuras investigaciones deben integrar muestras más amplias y otras medidas de variables (p. ej., violencia comunitaria, apoyo familiar) para ofrecer apoyo a los resultados encontrados.

\section{Referencias}

Arroyo-Pérez, Y. (2019). Perfil del funcionamiento ejecutivo y aspecto psicosociales de adolescentes varones intervenidos por el tribunal de menores de Puerto Rico (Tesis doctoral inédita). Universidad Carlos Albizu, Puerto Rico.

Asato, M., Sweeney, J., \& Luna, B. (2006). Cognitive processes in the development of TOL performance. Neuropsychologia, 44(12), 2259-2269. doi: 10.1016/j.neuropsychologia.2006.05.010

Bakar, E., Taner, Y., Soysal, A., Karakas, S., \& Turgay, A. (2011). Behavioral rating inventory and laboratory tests measure different aspects of executive functioning in boys: A validity study. Klinik Psikofarmakoloji Bülteni-Bulletin of Clinical Psychopharmacology, 21(4), 302-316. doi: 10.5455/ bcp.20111004014003

Barkley, R. (2012). Executive functions: What they are, how they work, and why they evolved. Guilford Press.

Barkley, R., \& Fischer, M. (2011). Predicting impairment in major life activities and occupational functioning in hyperactive children as adults: Self-reported executive function (EF) deficits versus EF tests. Developmental Neuropsychology, 36(2), 137-161. doi: 10.1080/87565641.2010.549877

Berg, E. (1948). A simple objective technique for measuring flexibility in thinking. The Journal of General Psychology, 39(1), 15-22. doi: 10.1080/00221309.1948.9918159

Bologna, E. (2014). Estimación por intervalo del tamaño 
del efecto expresado como proporción de varianza explicada. Revista Evaluar, 14(1), 43-66. Recuperado de https://revistas.unc.edu.ar/index.php/ revaluar

Blakemore, S., Burnett, S., \& Dahl, R. (2010). The role of puberty in the developing adolescent brain. $\mathrm{Hu}$ man Brain Mapping, 31(6), 926-933. doi: 10.1002/ hbm. 21052

Carlson, S., Zelazo, P., \& Faja, S. (2013). Executive function. En P. D. Zelazo (Ed.), The Oxford Handbook of Developmental Psychology. Volumen I: Body and mind (pp. 706-743). New York, NY: Oxford University Press.

Comesaña, A., Stelzer, F., \& Introzzi, I. (2017). Inhibición de borrado en adultos mayores: Aportes para la validación de una tarea. Revista Evaluar, 17(2), 143153. doi: 10.35670/1667-4545.v17.n2.18727

Delis, D., Kaplan, E., \& Kramer, J. (2001). Delis-Kaplan Executive Function System (D-KEFS): Examiner's manual. San Antonio, TX: The Psychological Corporation.

DePrince, A., Weinzierl, K., \& Combs, M. (2009). Executive function performance and trauma exposure in a community sample of children. Child Abuse \& Neglect, 33(6), 353-361. doi: 10.1016/j.chiabu.2008.08.002

Dorna-Pesquera, S., \& Caballero-González, H. (2010). Informe estadístico anual de las procuradurías de menores: Año Fiscal 2008 - 2009 comparativo con el 2007 -2008, departamento de justicia. Recuperado del sitio de Estadística del Gobierno de Puerto Rico: http://www.estadisticas.gobierno.pr/iepr/ LinkClick.aspx?fileticket=VXTAUK13saA\%3D\&tabid $=186$

Donders, F. (1969). On the speed of mental processes. Acta Psychologica, 30, 412-431. doi: 10.1016/00016918(69)90065-1

Field, A. (2018). Discovering Statistics Using IBM SPSS
Statistics ( $5^{a}$ ed.). London, UK: SAGE Publications.

Fuster, J. (2000). Executive frontal functions. Experimental Brain Research, 133(1), 66-70. doi: 10.1007/ s002210000401

García-Molina, A., Tirapu-Ustárroz, J., \& Roig-Rovira, T. (2007). Validez ecológica en la exploración de las funciones ejecutivas. Anales de Psicología, 23(2), 289-299. Recuperado de https://revistas.um.es/ analesps/index

Gioia, G., Isquith, P., Guy, S., \& Kenworthy, L. (2000). Behavior rating inventory of executive function: BRIEF. Odessa, FL: Psychological Assessment Resources.

Harvey, P. (2012). Clinical applications of neuropsychological assessment. Dialogues in Clinical Neuroscience, 14(1), 91-99. doi: 10.31887/ DCNS.2012.14.1/pharvey

Hoskin, R. (2012). The dangers of self-report. Science Brainwaves.

IBM Corp. (2019). IBM SPSS Statistics for Windows (Versión 26.0). [Software de cómputo]. Armonk, NY: IBM Corp.

Krikorian, R., Bartok, J., \& Gay, N. (1994). Tower of London procedure: A standard method and developmental data. Journal of Clinical and Experimental Neuropsychology, 16(6), 840-850. doi: 10.1080/01688639408402697

Ley No 88. (1986). Ley de Menores. Puerto Rico. Recuperado de www.lexjuris.com

Loewenstein, D., Argüelles, S., Bravo, M., Freeman, R., Argüelles, T., Acevedo, A., \& Eisdorfer, C. (2001). Caregivers' judgments of the functional abilities of the Alzheimer' disease patient: A comparison of proxy reports and objective measures. The Journals of Gerontology Series B: Psychological Sciences and Social Sciences, 56(2), 78-84. doi: 
Morales-Millán, et. al., Evaluar, 2021, 21(1), 70-72

10.1093/geronb/56.2.P78

Logan, G., \& Cowan, W. (1984). On the ability to inhibit thought and action: A theory of an act of control. Psychological Review, 91(3), 295-327. doi: 10.1037/0033-295X.91.3.295

Lugo-Gil, J., \& Tamis-LeMonda, C. (2008). Family resources and parenting quality: Links to children's cognitive development across the first 3 years. Child Development, 79(4), 1065-1085. doi: 10.1111/j.1467-8624.2008.01176.x

Maldonado-Belmonte, M. (2016). Adaptación del BRIEF (Behavior Rating Inventory of Executive Function) a población española y su utilidad para el diagnóstico del trastorno por déficit de atención-hiperactividad subtipos inatento y combinado. (Tesis doctoral). Recuperado de https://dialnet.unirioja. es

Mesulam, M. (2002). The human frontal lobes: Transcending the default mode through contingent encoding. En D. T. Stuss \& R. T. Knight (Eds.), Principles of frontal lobe function (pp. 8-30). New York, NY: Oxford University Press.

Mitchell, M., \& Miller, L. (2008). Prediction of functional status in older adults: The ecological validity of four Delis-Kaplan Executive Function System tests. Journal of Clinical and Experimental Neuropsychology, 30(6), 683-690. doi: $10.1080 / 13803390701679893$

Naglieri, J., \& Goldstein, S. (2013). Comprehensive Executive Function Inventory. North Tonawanda, NY: Multi-Health Systems.

Nęcka, E., Lech, B., Sobczyk, N., \& Śmieja, M. (2012). How much do we know about our own cognitive control? European Journal of Psychological Assessment, 28(3), 240-247. doi: 10.1027/1015$5759 / \mathrm{a} 000147$

Nelson, E., Leibenluft, E., McClure, E., \& Pine, D. (2005). The social re-orientation of adolescence: A neu- roscience perspective on the process and its relation to psychopathology. Psychological Medicine, 35(2), 163-174. doi: 10.1017/S0033291704003915

Nordvall, O., Jonsson, B., \& Stigsdotter-Neely, A. (2017). Self-reported and performance-based measures of executive functions in interned youth. Psychology, Crime \& Law, 23(3), 240-253. doi: 10.1080/1068316X.2016.1239725

Op den Kelder, R., Van den Akker, A., Geurts, H., Lindauer, R., \& Overbeek, G. (2018). Executive functions in trauma-exposed youth: A meta-analysis. European Journal of Psychotraumatology, 9(1), Artículo $\mathrm{N}^{\circ}$ 1450595. doi: 10.1080/20008198.2018.1450595

Patros, C., Tarle, S., Alderson, R., Lea, S., \& Arrington, E. (2019). Planning deficits in children with attention-deficit/hyperactivity disorder (ADHD): A meta-analytic review of tower task performance. Neuropsychology, 33(3), 425-444. doi: 10.1037/ neu0000531

Paulhus, D., \& Vazire, S. (2007). The self-report method. En R.W. Robins, R. C. Fraley \& R. F. Krueger (Eds.), Handbook of Research Methods in Personality Psychology (pp. 224-239). New York, NY: The Guildford Press.

Pérez-Salas, C., Ramos, C., Oliva, K., \& Ortega, A. (2016). Bifactor modeling of the Behavior Rating Inventory of Executive Function (BRIEF) in a Chilean sample. Perceptual and Motor Skills, 122(3), 757776. doi: 10.1177/0031512516650441

Raine, A. (2002). Biosocial studies of antisocial and violent behavior in children and adults: A review. Journal of Abnormal Child Psychology, 30(4), 311-326. Recuperado de https:/www.springer.com/journal/10802

Rathinabalan, I., \& Naaraayan, S. (2018). Effect of personal and school factors on juvenile delinquency. The Indian Journal of Pediatrics, 85(7), 569. doi: 10.1007/s12098-017-2566-z 
Reitan, R. (1958). Validity of the Trail Making Test as an indicator of organic brain damage. Perceptual and Motor Skills, 8(3), 271-276. Recuperado de https://journals.sagepub.com/home/pms

Roberts, S., \& Apaza, R. (2011). Funciones ejecutivas, atención y conducta. Revista de Investigación UCSP, 2(2), 7-28. Recuperado de http://repositorio.unsa.edu.pe

Rueda, M., Cómbita, L., \& Pozuelos, J. (2016). Childhood and adolescence. En T. Strobach \& J. Karbach (Eds.), Cognitive training: An overview of features and applications (pp. 33-44). doi: 10.1007/978-3319-42662-4_4

Schore, A. (2001). Effects of a secure attachment relationship on right brain development, affect regulation, and infant mental health. Infant Mental Health Journal Publication of The World Association for Infant Mental Health, 22(1-2), 7-66. Recuperado de https://onlinelibrary.wiley.com/journal/10970355

Sedó, M. (2004). '5 Digit Test': A multilinguistic non-reading alternative to the Stroop test. Revista de Neurología, 38(9), 824-828. doi: 10.33588/ rn.3809.2003545

Shallice, T. (1982). Specific impairments of planning. Philosophical Transactions of the Royal Society of London. Serie B: Biological Sciences, 298(1089), 199-209. doi: 10.1098/rstb.1982.0082

Sowell, E., Thompson, P., Holmes, C., Batth, R., Jernigan, T., \& Toga, A. (1999). Localizing age-related changes in brain structure between childhood and adolescence using statistical parametric mapping. Neuroimage, 9(6), 587-597. doi: 10.1006/ nimg. 1999.0436

Spooner, D., \& Pachana, N. (2006). Ecological validity in neuropsychological assessment: A case for greater consideration in research with neurologically intact populations. Archives of Clinical
Neuropsychology, 21(4), 327-337. doi: 10.1016/j. acn.2006.04.004

Steinberg, L. (2010). A dual systems model of adolescent risk-taking. Developmental Psychobiology, 52(3), 216-224. doi: 10.1002/dev.20445

Stroop, J. (1935). Studies of interference in serial verbal reactions. Journal of Experimental Psychology, 18(6), 643-662. doi: 10.1037/h0054651

Suchy, Y. (2015). Executive functioning: A comprehensive guide for clinical practice. New York, NY: Oxford University Press.

Teicher, M., \& Samson, J. (2016). Annual research review: Enduring neurobiological effects of childhood abuse and neglect. Journal of Child Psychology and Psychiatry, 57(3), 241-266. doi: 10.1111/ jcpp. 12507

Thierry, A., Glowinski, J., Goldman-Rakic, P., \& Christen, Y. (Eds.). (1994). Motor and cognitive function of the prefrontal cortex. Berlin, Alemania: Springer.

Toplak, M., West, R., \& Stanovich, K. (2013). Practitioner review: Do performance-based measures and ratings of executive function assess the same construct? Journal of Child Psychology and Psychiatry, 54(2), 131-143. doi: 10.1111/jcpp.12001

Vasterling, J., \& Brailey, K. (2005). Neuropsychological findings in adults with PTSD. En J. Vasterling \& C. Brewin (Eds.), Neuropsychology of PTSD: Biological, Cognitive, and Clinical Perspectives (pp. 178-207). New York, NY: The Guilford Press.

Vélez-Pastrana, M., González, R., Rodríguez-Cardona, J., Purcell-Baerga, P., Alicea-Rodríguez, Á., \& Levin, F. (2016). Psychometric properties of the Barkley Deficits in Executive Functioning Scale: A Spanish-language version in a community sample of Puerto Rican adults. Psychological Assessment, 28(5), 483-498. doi: 10.1037/pas0000171

Zanetti, O., Frisoni, G., Rozzini, L., Bianchetti, A., \& Tra- 
bucchi, M. (1998). Validity of Direct Assessment of Functional Status as a tool for measuring Alzheimer's disease severity. Age and Ageing, 27(5), 615-622. doi: 10.1093/ageing/27.5.615

Zelazo, P., Carter, A., Reznick, J., \& Frye, D. (1997). Early development of executive function: A problem-solving framework. Review of General Psycholgy, 1(2), 198-266. doi: 10.1037/10892680.1.2.198

Zysset, A., Kakebeeke, T., Messerli-Bürgy, N., Meyer, A., Stülb, K., Leegee-Aschmann, C. S., \& Jenni, O. (2018). Prediction of executive functions in preschoolers: Findings from the SPLASHY study. Frontiers in Psychology, 9. doi: 10.3389/ fpsyg. 2018.02060 